\title{
Study on the flexible Rayleigh waves comb transducer with non-equal interval based on PZT
}

\author{
Huamin Zhao ${ }^{1, \text { a }}$, Cunfu He $\mathrm{H}^{1, \mathrm{~b}}$, Lv Yan ${ }^{1, \mathrm{c}}$ \\ ${ }^{1}$ College of Mechanical Engineering and Applied Electronics Technology, \\ Beijing University of Technology, Beijing, 100124, China \\ azhaohuamin909@163.com, 'bhecunfu@bjut.edu.cn, 'Ivyan@bjut.edu.cn
}

Keywords: Rayleigh waves transducer, Flexible, Comb

\begin{abstract}
In order to detect the defects of inner and outer surfaces of thick-walled pipes, a Rayleigh waves flexible transducer with non-equal interval comb is designed. Firstly, an optimization design method based on the concept of mutual admittance is proposed, and the amplitude frequency characteristics of Rayleigh waves transducer with equal interval comb are optimized. The influence of the key parameters such as the array element interval, the width of the array element and the number of elements on the waveform and amplitude of the received signal are analyzed by using the finite element method. Based on the analysis of the mutual admittance principle and time frequency analysis, it is determined that the optimal element interval is equal to $\lambda_{R}$, the element width is $\lambda_{R} / 2$, and the elements number is 5. Finally, based on the conclusion of equal interval transducer, the non-equal interval comb transducer is designed to obtain a good Rayleigh wave signal, which provided a good foundation for the practical manufacture and experiment of the transducer.
\end{abstract}

\section{Introduction}

Thick-walled pipe is a key component of thermal power generating set, which plays an important role in the safety and normal operation of power plant. However, some of the thick-walled pipes are often uneven in metallographic structure, poor shape tolerance control, processing defects, pits, cracks and other phenomena [1]. So a method for rapid detection of surface defects in thick-wall pipes is needed.

The surface and internal defects of the work piece are detected quickly, completely, widely by the technology of ultrasonic guided waves [2]. Rose et al. used Sokolinskii type to make guided wave transducer [3], and they obtained good results by adjusting the element interval on the board to stimulate a specific mode of guided wave. Borigo and Koduru et al. [4] established a mathematical model for annular comb transducer on board. The effects of time and phase delay on modal selection are studied, and the purpose of modal control in all directions is achieved. The commonly used flexible comb piezoelectric transducer is mainly PVDF piezoelectric film, but it is poor compared with the PZT incentive performance. Based on the above requirements, a Rayleigh waves transducer with flexible non-equal interval comb broadband is studied based on PZT for thick-walled pipe detection, transducer rendering shown in Fig. 1.

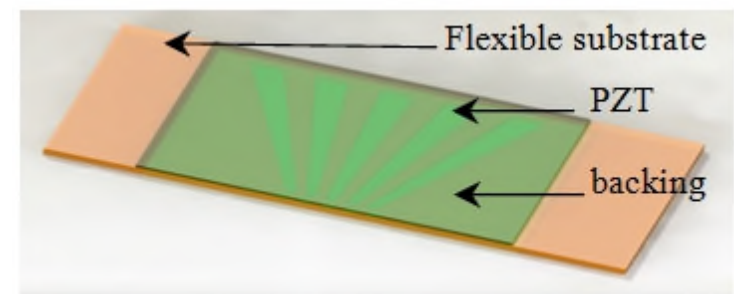

Fig. 1 Comb-shaped broadband transducer rendering. 


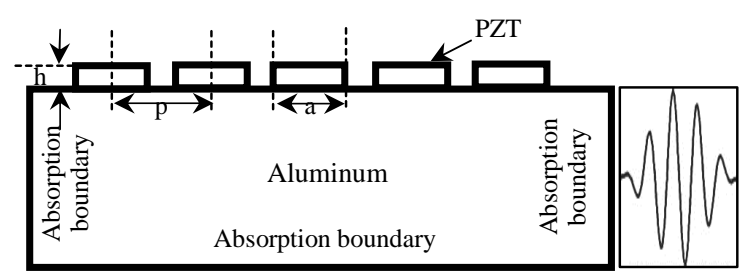

Fig. 2 Two dimensional simulation model and excitation signal

\section{Parameters Optimization of Equal Interval Comb Transducer}

In order to determine the optimum parameter of the equal interval Rayleigh wave transducer, the PZFlex software was used to analyze the influence of the distance $p$, the width $a$ and the number of the piezoelectric plate on the performance of the transducer. Each piezoelectric element of the comb transducer is one array element (comb tooth). $500 \mathrm{kHz}$ sine wave modulating by 5 cycle Hanning window is used as the simulation signal. The width and thickness of model aluminum plate are $600 \mathrm{~mm}$ and $200 \mathrm{~mm}$, respectively. The grid size is the Rayleigh wavelength of $1 / 20$. The left and right and lower boundaries of the aluminum plate are set to the absorbing boundary. Simulation model and simulation signal are shown in Fig. 2.

Ballandras [5] analyzed the periodic structure of 2-2 connected composites based on the principle of mutual admittance. The mutual admittance theory can be found in the article. The comb Rayleigh wave transducer can be optimized by calculating the admittance changes of the other elements caused by the vibration of one element. The smaller the resulting admittance, the smaller the mutual influence between the elements, the better the transducer excitation.

First, the width of the array is $\lambda_{R} / 2$, and the number of elements is equal to 5 . The proportion coefficient of the element interval and excitation wavelength is optimized. The ratio of the array element interval to the excitation wavelength is $0.6,0.7,0.8,0.9,1.0,1.1$ and 1.2 respectively. The admittance values of the five element array transducers are calculated respectively. Admittance value of elements of the comb transducer is shown in Fig. 3 with the proportional coefficient of 0.6, $1.0,1.2$. At the same time, the maximum admittance value of the five elements under each scale factor is extracted, and the amplitude of the admittance is normalized according to the scale factor of the element interval and excitation wavelength, as shown in Fig. 4.

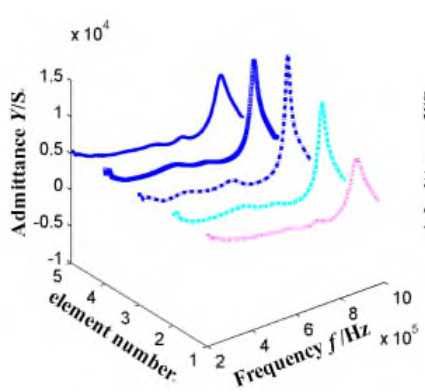

(a) 0.6

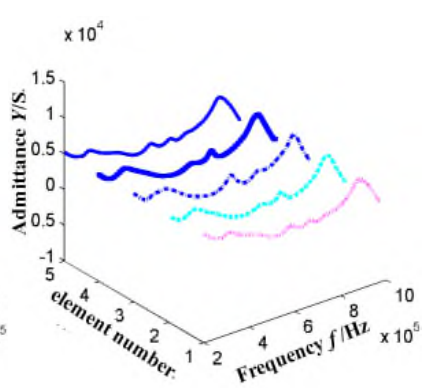

(b) 1.0

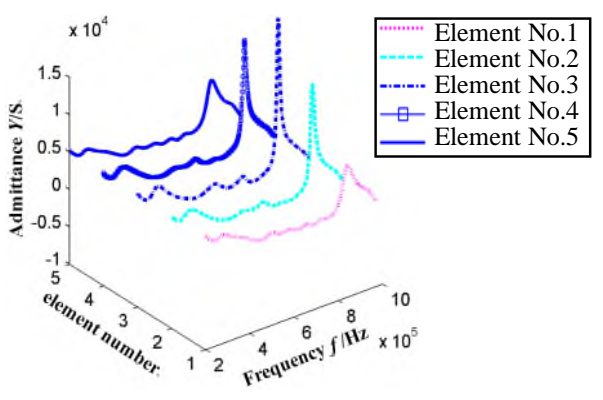

(c) 1.2

Fig. 3 3-D admittance of the different ratio of element spacing versus the excitation wavelength.

The normalized amplitude of the admittance decreases and then increases with the increase of the ratio of the element interval to the excitation wavelength, as seen in Fig. 4. When the scale factor is in the range of 0.8 to 1.0 , the amplitude of the admittance is smaller. This indicates that mutual interference between the elements is relatively small. In addition, the normalized amplitude of the received signal is shown in Fig. 6, when the ratio coefficient of the interval and excitation signal is 1 , the amplitude is large. So the element interval is designed to be equal to the excitation wavelength.

When optimize the width of the element, the array interval is fixed as $\lambda_{R}$ and the number of elements is 5 . The time domain waveform is analyzed when the element width a is $\lambda_{R} / 4, \lambda_{R} / 2,3 \lambda_{R} / 4$ respectively. As shown in Fig. 5. From the time-domain waveforms, it can be concluded that, when 
the interval is $\lambda_{\mathrm{R}} / 2$, the Rayleigh wave time-domain signal tailing is significantly smaller than the Rayleigh wave signal with $a=\lambda_{\mathrm{R}} / 4$ and $3 \lambda_{\mathrm{R}} / 4$, respectively. Therefore, when the element width is $\lambda_{\mathrm{R}} / 2$, it is the optimal element width.

The element interval is fixed as $\lambda_{R}$, and element width is $\lambda_{R} / 2$ to optimize the element number $n$. The number of elements varies from 3 to 10, respectively. The relationship among the amplitude, the bandwidth and the number of elements of the Rayleigh wave signal is analyzed. As shown in Fig. 6, the amplitude of the Rayleigh wave signal increases first and then decreases with the increase of the number of elements. The amplitude of the signal is greatest when the element $\mathrm{n}$ is equal to 5. As shown in Fig. 6, the signal bandwidth decreases as the number of elements increases. As shown in Fig. 7, the time domain waveform gradually widens as the number of elements increases, and the tailing begins to increase, and it reduces the resolution of the transducer. Too much element can increase the size of the transducer. After a comprehensive analysis, it determines the $\mathrm{n}$ is 5 for the best.

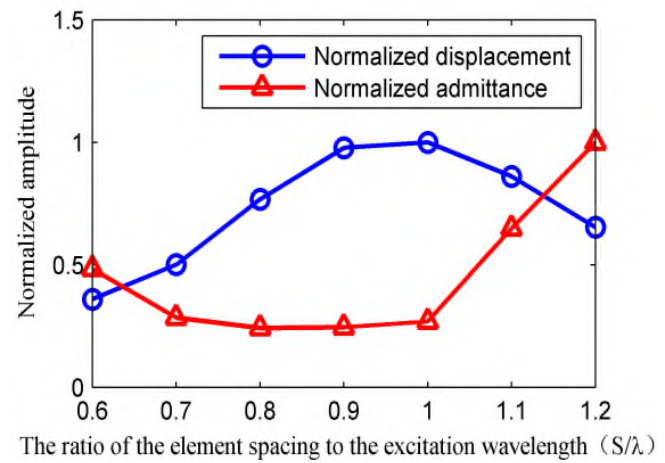

Fig. 4The admittance normalized amplitude of the different ratio of element interval versus the excitation wavelength.
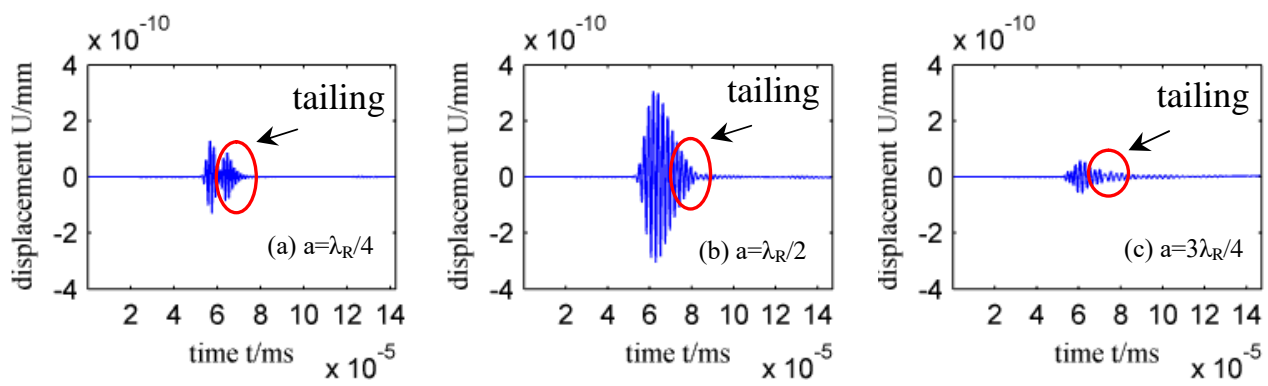

Fig. 5 The time domain waveform of different element width.

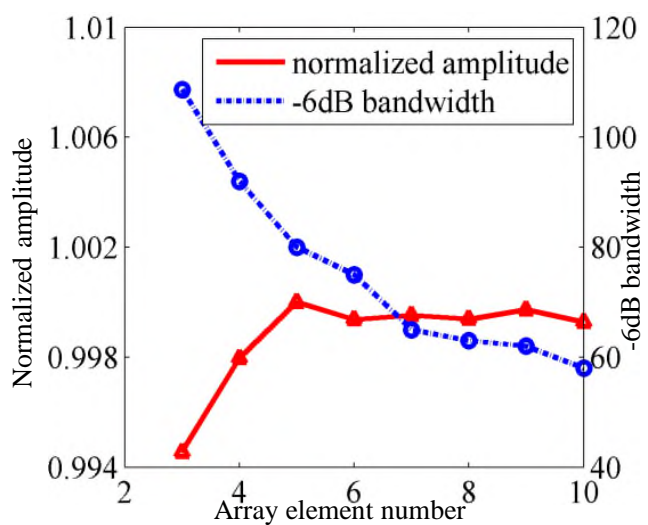

Fig. 6 The normalized amplitude and the $-6 \mathrm{~dB}$ bandwidth of different element number. 

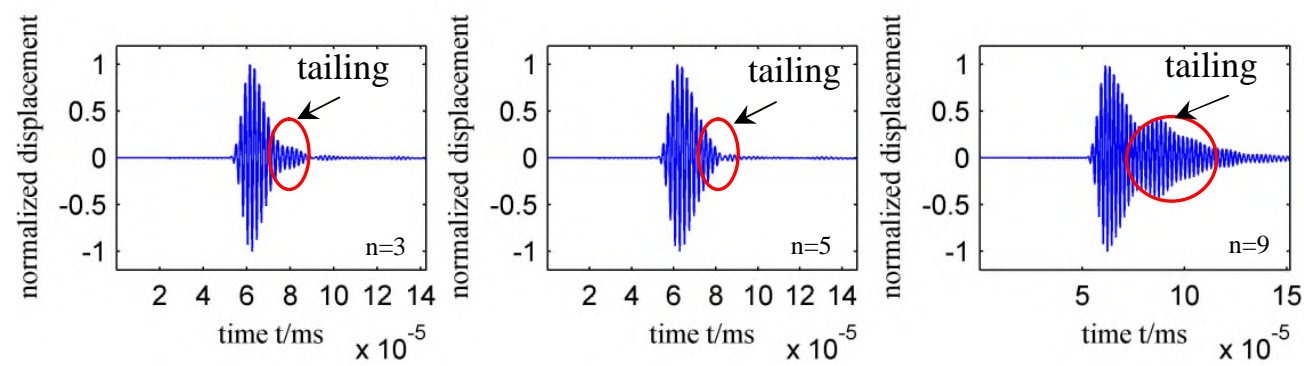

Fig. 7 The time domain waveform of different element number.

By analyzing the width of element interval and the number of elements, it is finally determined that $p$ equals to $\lambda_{\mathrm{R}}$, equals to $\lambda_{\mathrm{R}} / 2$, and $n$ equals to 5 is the optimal transducer parameters.

\section{Non-Equal Interval Transducer Design}

The non-equal interval transducer is designed according to the results of equal interval comb transducer optimization. The shape of the transducer is shown in Fig. 8. The element width of the comb transducer is different corresponding to different excitation frequency of half wavelength, respectively. The interval of the comb transducer is also varied according to the wavelength of the Rayleigh wave which is required to be excited. As shown in Fig. 8, the excitation frequency of the non-equal interval transducer is designed to range from $500 \mathrm{kHz}$ to $700 \mathrm{kHz}$. $500 \mathrm{kHz}$ Rayleigh waves wavelength is equal to $6 \mathrm{~mm}$, so the transducer element at the widest point $p_{1}$ is equal to $6 \mathrm{~mm}$, the widest array element width $a_{1}$ is equal to half of wavelength $3 \mathrm{~mm}, p_{2}$ is set to $4.3 \mathrm{~mm}$ in $700 \mathrm{kHz}$, $a_{2}$ is $2.15 \mathrm{~mm}$. The middle part changes in turn. The comb element length $L$ and the thickness are determined by impedance analysis of the PZT. By adjusting the length $L$ and PZT thickness, and apply a backing to achieve the transducer's broadband. So that the transducer excitation frequency ranges of $500 \mathrm{kHz}-700 \mathrm{kHz}$. By PZflex software impedance analysis, when the thickness of the transducer element is $1 \mathrm{~mm}$, length $L$ is $15 \mathrm{~mm}$, it meets the requirements. The impedance analysis results of the left-most element are shown in Fig. 9. As can be seen from Fig. 9, the center frequency of the transducer element PZT is from $500 \mathrm{kHz}$ to $700 \mathrm{kHz}$. The impedance analysis results of other elements are also similar.

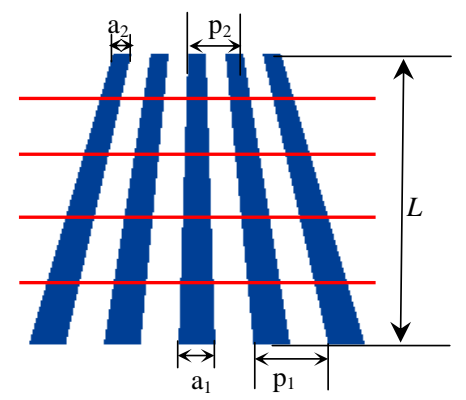

Fig. 8 Sketch map of non-equal interval comb transducer.
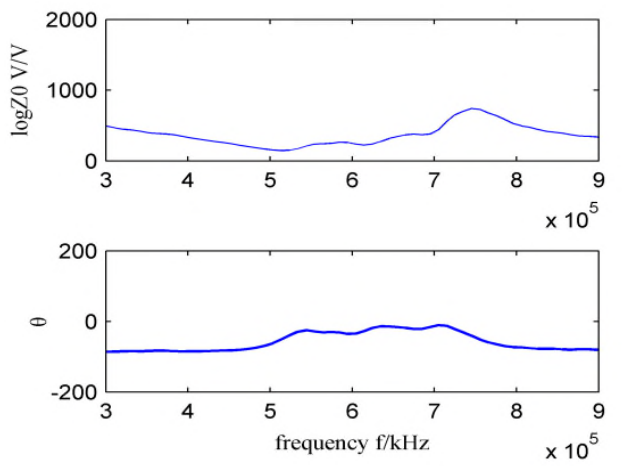

Fig. 9 Impedance analysis of No.1 array element. 
The transducer is designed and simulated according to the size of the above analysis using Hanning window signal excitation with $500 \mathrm{kHz}, 600 \mathrm{kHz}, 700 \mathrm{KHz}$, respectively. The time-domain waveform of receiving is shown in Fig. 10. From the time domain waveform can be seen, the signal tailing of the three frequency excitation is very small. It can ensure that non-equal interval transducer has a better resolution to meet the detection requirements of defects.
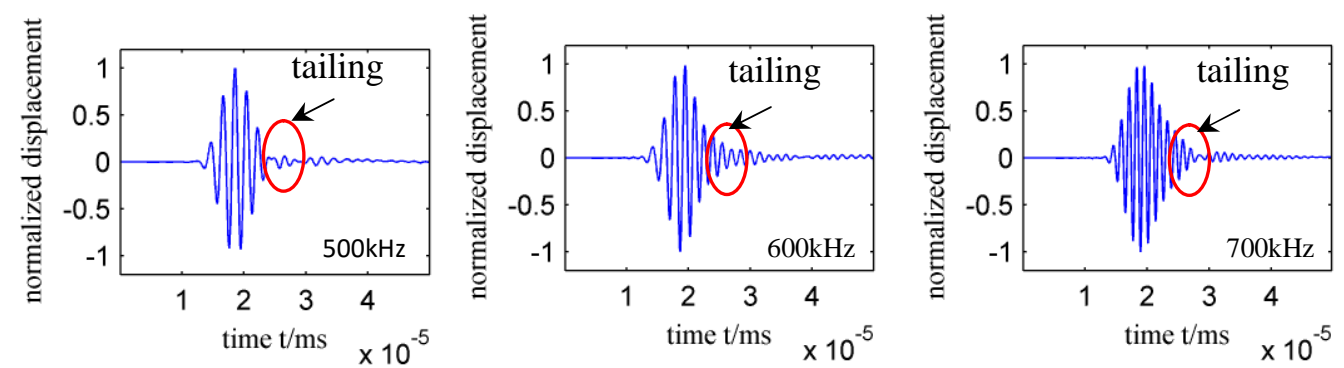

Fig. 10 Time domain waveform of different frequency Rayleigh waves.

\section{Conclusion}

Through simulation analysis the best parameters of comb transducer are obtained. When the array element interval is $\lambda_{\mathrm{R}}$, the element width is $\lambda_{\mathrm{R}} / 2$ and the number of elements is 5 , the energy of the excited Rayleigh wave signal is the most concentrated and the tailing is the smallest. Finally, based on the equal interval optimization results, the non-equal interval transducer can generate good Rayleigh wave signal by $500 \mathrm{kHz}-700 \mathrm{kHz}$ excitation, which provides the theoretical basis for the post-production transducer.

\section{Acknowledgement}

In this paper, the research was sponsored by the National Natural Science Foundation of China (Key Program) (Project No. 51235001).

\section{References}

[1] Z. Liu Experimental research on circumferential guided wave scanning imaging of thick wall pipes based on continuous wavelet transform. J. Mech. Eng. 49(2) (2013) 14-19.

[2] A. Demma, P. Cawley, M. Lowe, et al. The reflection of the fundamental torsional mode form cracks and notches in pipes. J. Acoust. Soc. Am. 114(2) (2003) 611-625.

[3] J. L. Rose, S. P. Pelts, M. J. Quarry. A comb transducer for mode control in guided wave NDE. Proc. IEEE Ultrasonics Symposium, 2 (1997) 1033-1036.

[4] J. L. Rose. Standing on the shoulders of giants: an example of guided wave inspection. Mater. Evaluat. 60(1) (2002) 53-59.

[5] S. Ballandras, M. Wilm, P. Edoa, et al. Finite- element analysis of periodic piezoelectric transducers. J. Appl. Phys. 93(1) (2003) 702-711. 